\title{
Tecnología y globalización en el transporte marítimo
}

Technology and globalization in maritime transport

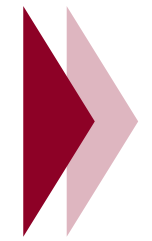

José Alberto Reyes García

Msc. en Transporte Marítimo. Universidad Nacional Experimental Marítima del Caribe. Venezuela Lic. en Estudios Internacionales. Universidad Central de Venezuela. Abogado. Universidad Central de Venezuela. Miembro Titular del Instituto Iberoamericano de Derecho Marítimo.

Correo electrónico: jareyesg@gmail.com 


\section{Resumen}

En el mundo globalizado y actual donde el comercio crece a un ritmo acelerado, se hace necesario cada vez más la presencia de mejoras en el sector transporte; ya que del mismo depende la perfecta comercialización y distribución de los rubros que se importen o exporten por parte de cualquier país, de acuerdo a sus necesidades comerciales y financieras, en cuanto a la obtención de ingresos, bienes o s ervicios. A pesar de las actuales crisis financieras y económicas globales, el sector de la industria marítima, a través de su comunidad de socios y trabajadores, evalúan al máximo las mejoras necesarias para mitigar dicho efecto, para de esta manera poder prevenir que el sector marítimo -como prestador de servicios- se vea lo menos posible afectado por la caída en la demanda y los niveles de precios, razón por la cual se efectúa dicha investigación bajo la modalidad documental y análisis crítico, debido a que la técnica de recolección de información que se utilizó para realizar el estudio, estuvo relacionada con la revisión de fuentes documentales tales como: libros, revistas, informes, periódicos, convenios, acuerdos, medios electrónicos, entre otros. Dicho artículo de investigación se realizó con el principal basamento de generar resultados producto del análisis y discusión en las actuaciones de estrategias orientadas a la aplicación de tecnología y globalización en el transporte marítimo con la finalidad que sirva de fuente de información a personas interesadas y vinculadas al área de la línea de investigación en negocio marítimo.

Palabras clave: tecnología, globalización, política comercial, marina mercante, integración económica, transporte marítimo.

\section{Abstract}

In the globalized and current world where trade grows at an accelerated pace, the presence of improvements in the transport sector is increasingly necessary; since it depends on the perfect marketing and distribution of the items that are imported or exported by any country according to their commercial and financial needs in terms of obtaining income, goods or services. In spite of the current global financial and economic crises, the maritime industry sector through its community of partners and workers evaluate to the maximum the improvements necessary to mitigate this effect, in order to prevent the maritime sector - as a provider of services- is seen as little as possible affected by the fall in demand and price levels, which is why this research is carried out under the documentary and critical analysis modality, due to the information collection technique that was used to perform The study was related to the review of documentary sources such as: books, magazines, reports, newspapers, agreements, agreements, electronic media, among others. Said research article was carried out with the main basis of generating results of the analysis and discussion in the actions of strategies oriented to the application of technology and globalization in maritime transportation with the purpose that it serves as a source of information to interested people and linked to the area of the research line in maritime business.

Keywords: technology, globalization, commercial policy, merchant navy, economic integration, maritime transport.

\section{Introducción}

Desde la historia de la evolución del hombre moderno y su forma de organización, los Estados que forman la sociedad internacional naciente, fueron tomando medidas de acuerdo a sus necesidades para planificar y desarrollar las herramientas necesaria que fortalecieran la convivencia humana, precisando cuáles eran sus respectivas competencias sobre sus diversos espacios marítimos ya que a través de los mismos surge la necesidad de permitir el avance y comunicación del hombre en el globo terráqueo. Según Wiener (1948) indica que: "la sociedad solo puede comprenderse al estudiar 
su intercambio de mensajes y sus instrumentos de comunicación, en cuyo desarrollo futuro la comunicación entre el hombre y las máquinas, entre las máquinas y el hombre, y entre las propias máquinas, tendrá una importancia cada vez mayor". En la actualidad, se han incrementado y diversificado los usos del medio marino, impulsados por el crecimiento demográfico y económico, por el desarrollo de la de tecnología, y los intereses que los Estados tienen en ellos, ocasionando que se haga compleja la ordenación jurídica de los mares y océanos, apareciendo otros espacios marítimos como: la plataforma continental, la zona económica exclusiva, la zona internacional de los fondos marítimos, entre otros.

Razón por la cual, existe la promoción del comercio, la integración regional y el desarrollo de una sociedad de la información, que hoy en día son aspectos cada vez más importantes de las políticas de desarrollo mundial, bien sea a nivel de gestión, seguridad, calidad y promoción del comercio, tanto para los gobiernos individuales como para la sociedad internacional. En la medida en que estos se reconozcan como motivos de preocupación independiente y a su vez mutuos, las tecnologías de la información y las comunicaciones (TIC) posibilitan, promueven y facilitan el comercio internacional, la integración regional y el desarrollo económico.

Debido a que la información ha desempeñado un papel fundamental a través de la historia y la posibilidad de compartirla, mediante la comunicación, continúa asombrando a la humanidad. El intercambio de información determina la conducta del ser humano, al punto que lingüistas y biólogos sostienen que el almacenaje de información por medio de diversas técnicas, como el arte, el lenguaje o las herramientas, fue la fuerza impulsora que llevó a los seres humanos a convertirse en la especie dominante del planeta. Sin desconocer la importancia de la información para la vida y la existencia humana, el siguiente análisis teórico analiza desde una perspectiva económica y social la importancia de la sociedad de la información como un tipo de sociedad en el que la captación, almacenamiento, transmisión y computación de la información como las acciones socio económicas más importantes para preservar la vida humana y generar confianza en el intercambio de mercancías mediante el uso del Transporte Marítimo. La economía y el transporte, hoy en día es un sector fuertemente golpeado por los males o síntomas que generaron la crisis internacional en las economías de los países más desarrollados, la que tomo fuerte expresión a mediados del año 2008 y se extendió a nivel global desde principios del 2009, para este caso se observaron señales de sobre-tonelaje y la mayoría de los proyectos de expansión fueron revisados o suspendidos; los precios de la actividad presentaron fuertes caídas, la proporción de buques amarrados sin trabajo aumentó, generando grandes preocupaciones en dicho sector (Sánchez, 2009).

Debido a que el 66\% de la superficie del mundo está cubierta por mares y océanos, el transporte marítimo tiene una importancia geoestratégica, unido a ello también es evidente que a través de buques es más eficaz la movilización de grandes volúmenes de carga en cuanto a su distribución y costos.

\section{Antecedentes}

Años antes de cristo, se descubre que existen las costas orientales del Mediterráneo, por lo que suele mencionarse que los primeros navegantes que dieron origen a las nacientes actividades culturales y comerciales económicas del mundo fueron los de la antigua Grecia, del Imperio Romano y en el antiguo Egipto. En la última etapa de la edad media, la construcción naval fue evolucionando, y aparecen los buques de vela, ya que este tipo de embarcaciones tenían la ventaja de garantizar una mayor velocidad en sus recorridos de largas distancias, pero con la desventaja de tener una insuficiente capacidad de carga. Ante la necesidad imperante de desarrollar un buque que permitiera una mayor capacidad de carga, aparece en la mitad del siglo XVI un prototipo de buque llamado galeón con la 
característica de poseer bodegas para cargar provisiones y mercancías y otra embarcación llamada la carabela que a diferencia de la misma fue más utilizada en los tiempos de los descubrimientos iniciados en las rutas entre Europa - América o Europa - Asia mediante el cabo de Buena Esperanza.

En lo que se refiere a la historia de la República Bolivariana de Venezuela, podemos citar que el primer presidente en interesarse en la implementación de la industria naval en el país, fue, de acuerdo a la gaceta número 9.551 de fecha martes 29 de agosto de 1905, el presidente venezolano Cipriano Castro quien ordenó en su artículo 1: la construcción de un dique carenero en el paraje más adecuado de Puerto Cabello.

Durante los siglos siguientes, la construcción naval evoluciona, dándose cambios importantes en el sistema de propulsión, pasando de buques de velas a buques a vapor, lo que hizo que el hombre tomara conciencia de la necesidad del uso de nuevos materiales y herramientas en la construcción de embarcaciones por las siguientes razones:

a. Evoluciona por la necesidad que tenía el hombre de preservar su vida

- El medio acuático le garantizaba soluciones

- Nuevas fronteras

- Alejarse del peligro

- Medio de transporte y superación en su forma de vida

- El hombre desde la historia ha desarrollado la creatividad como ser pensante, por lo cual ha hecho uso de diferentes materiales para demostrar sus habilidades

- La madera: históricamente ha sido la materia prima proporcionada por el medio.

b. Otro factor, fue permitir el desarrollo y comunicación del hombre en el globo terráqueo.

El concepto de sociedad de la información fue creado por Machlup en (1962), cuya conclusión fue que el número de personas dedicadas al manejo y procesamiento de información era mayor que el de quienes realizaban tareas físicas. Otros autores, como Drucker (1969) y Bell (1973), destacaron que el conocimiento sería el principal factor de generación de riqueza en la sociedad del futuro; por su parte, Masuda (1981) analizó el papel de la información como el principal componente de este proceso y las condiciones tecnológicas necesarias para su desarrollo. La tecnología no se puede reducir a un conjunto de diseños o planos que definen un producto; por el contrario, consiste en actividades orientadas a la solución de problemas, que incorporan formas tácitas de conocimiento mediante procesos individuales o institucionales.

El inicio de la formación de una organización mundial, interesada en conseguir un sistema global único de comunicaciones (en principio marítimas), podemos encontrarlo en la WARC de 1971, cuando se asignaron bandas de frecuencias de funcionamiento al Servicio Móvil Marítimo por Satélite (GMSS), y países con gran movimiento náutico, empezaron a comprender la necesidad de coordinar esfuerzos y aproximar posiciones para poder actuar de forma conjunta, lo que requirió numerosos encuentros y estudios a todos los niveles que acabaron en la constitución de Inmarsat, con un modelo parecido al adoptado por Intelsat. WARC (Warminster Amateur Radio Club), es un club de radio de propósito general cuyos miembros hacen especial hincapié en proveer soporte de comunicaciones a servicios públicos; el club fue fundado en 1964 y en la actualidad cuenta con 110 miembros.

Una conferencia en 1976 concluyó con la Convención y el Acuerdo Operacional de la Organización Internacional de Satélites Marítimos (Inmarsat). Ambos instrumentos entraron en vigor en julio de 1979, tras alcanzarse la firma por 26 Estados (partes) que cubrieron el 95\% de las participaciones de inversión previstas. El origen de la constitución de una compañía que estableciera un sistema global de comunicaciones vía satélite, para perfeccionar las comunicaciones marítimas, puede establecerse en febrero de 1976, fecha en que se inauguró el sistema comercial Marisat, promovido por un consorcio de empresas norteamericanas. 
Utilizaba uno de los satélites destinados a la marina de aquel país. En 1979 ya funcionaba el primer sistema de comunicaciones marítimas comerciales por satélite de cobertura global, con un satélite sobre cada uno de los océanos: Atlántico, Pacífico e Índico. Dado que la ESA (Agencia Espacial Europea) estaba desarrollando satélites experimentales para el servicio marítimo, redefiniéndose algunos de sus parámetros para hacerlos compatibles con el sistema Marisat de los americanos, estableciéndose en ese momento las bases de una fructífera colaboración internacional que acabó en la formación de Inmarsat, (International

Maritime Satellite Organization), la cual se crea en 1979, siendo una organización internacional que operaba un sistema mundial de comunicaciones móviles por satélite y funcionaba a modo de cooperativa. En un principio, se fundó para mejorar las comunicaciones marítimas con objeto de incrementar la seguridad en el mar. Actualmente, además de suministrar servicios de telefonía y transmisión de datos a embarcaciones y plataformas marítimas, aporta también servicios para la comunidad aeronáutica y para los móviles terrestres. De los 26 países que participaron en su constitución (entre ellos España), ha pasado a tener hoy en día 79 países miembros, de los cuales Estados Unidos cuenta con la mayor parte (alrededor de un 23\%), el Reino Unido y Noruega poseen el $11 \%$ y el $10.5 \%$ respectivamente.

\section{Evolución de la construcción naval}

En la moderna construcción naval se hace uso de diferentes materiales (sin embargo, el acero tiene el predominio sobre el resto). Por razones de economía de peso, en los buques se emplean aleaciones de aluminio, en sitios como: "los lugares altos del casco". El plástico es usado en embarcaciones de recreo, botes salvavidas y pesqueros de pequeño porte. Actualmente la madera es utilizada en menor porcentaje, pero normalmente circunscrita para barcos pesqueros, por lo que podemos decir que la construcción naval es el vaso flotante que se utiliza para la comunicación comercial mundial.

La madera en la construcción naval: su principal característica como medio de flotación es la ligereza, que le permite flotar sobre el agua, además está la facilidad con la que se puede trabajar a través del uso de herramientas, si se quiere primitiva. Fue utilizada por el hombre no solamente para la fabricación del casco, también la utilizó para la arboladura y vergas de los buques. Por el aumento del tamaño de estos, la madera de los árboles presentó problemas por las dimensiones de las piezas que se necesitaban, por lo que se desarrollaron nuevas técnicas, como el contra enchapado: en que las chapas de maderas van superpuestas con resinas, el encolado se hace en frío, utilizando colas especiales resistentes al agua de mar.

Desventajas del uso de la madera: se construye sin cálculo alguno (no pueden fijarse coeficientes de elasticidad y resistencia con debida precisión), además, se presentan grietas producidas por heladas, heridas de rayos, podredumbre, carcoma, distribución de fibras no uniformes. La madera con nudos, vetas atravesadas o grietas se trabaja muy mal y supone un riesgo para la seguridad de la estructura.

El ferro cemento en la construcción naval: su utilización se inicia durante la guerra de 1914-1918 con la construcción de algunos buques de hormigón armado (ninguno navega en la actualidad). Estos barcos fueron de línea moderna, tenían 11 compartimientos de las cuales 7 eran bodegas de carga, cámara de máquinas, tangue de combustibles y los 2 piques, desarrollaban una velocidad de 10 nudos y su tripulación era de 29 hombres.

Desventajas del ferro cemento: elevado precio y dificultad para conseguir perfectamente la estanqueidad. El sufrimiento de fisuras que afectan la impermeabilidad y su excesivo peso.

El hierro en la construcción naval: a principios del siglo XIX se aplica este material en la construcción de barcos, con el uso del mismo, se introdu- 
ce la hélice, demostrando su superioridad sobre la rueda de paletas que era el medio propulsor más utilizado para la época. Según Castillo (1983) el primer vapor de hierro fue el "Aarón Mamby" que tenía 25,3 mts de eslora y 5,6 mts de manga, demostrando las cualidades que le preservaba la experiencia en el mar.

El acero en la construcción naval: es el más utilizado actualmente para la fabricación de modernos buques. Tiene diferentes formas de acuerdo a la necesidad y los requerimientos del armador: el acero forjado, fundido y de alta resistencia, utilizado en la construcción naval es fabricado por acerías aprobadas por las sociedades de clasificación, bajo los procedimientos indicados por dichas sociedades.

Ventajas del uso del acero: mayor impermeabilidad, mayor duración, facilidad de construcción y reparación, mayor seguridad frente a siniestros, menor peso del casco, menor volumen de soldadura, mayor peso muerto a igualdad de dimensiones principales y por último secciones de planchas y perfiles más ligeras.

Importancia y evolución de las TIC en el transporte marítimo: China y el comercio electrónico como intercambio comercial: en cuanto a sus mercados informáticos, a) Los mercados de equipos y software han crecido rápidamente, a una tasa anual de más del $12 \%$ y más del $35 \%$, respectivamente. No obstante, en comparación con la escala económica general de China, el valor de las ventas es bastante bajo, b) Los equipos informáticos representan más del 70\% de la participación en el mercado, mientras que al software y los servicios de información corresponden solo el $11 \%$ y el 16\%, respectivamente, c) La recolección de información y la publicidad de la empresa son los dos principales usos reales de Internet, pero son varias las empresas que también realizan transacciones de comercio electrónico en línea, d) La aplicación de las TIC y del comercio electrónico en las empresas todavía está en su fase inicial, pero la popularidad de estas tecnologías aumenta con rapidez.
En cuanto al uso e implementación de nuevas herramientas tecnológicas: a) Una encuesta realizada en el 2001 reveló que el 89\% de las empresas usaban Internet. Esta cifra aumentó al 98,6\% en la encuesta del 2002, b) La línea telefónica y el modem son los principales métodos de conexión. En el 2001, el 50\% de las empresas utilizaban estos dos métodos, pero esta proporción disminuyó al 43,8\% en el 2002, c) El uso de líneas dedicadas aumentó del $25 \%$ en el 2001 al 36,8\% en el 2002. El uso de ADSL se incrementó vertiginosamente del $2 \%$ en el 2001 al 26,5\% en el 2002. La red digital de servicios integrados (ISDN) quedó en cuarto lugar, con el $16 \%$.

El negocio electrónico en China: los sitios web de comercio electrónico que funcionan en forma continua aumentaron de 575 en el 2.000 a 1.533 en el 2002. El volumen del comercio electrónico aumentó 3,5 veces, de 77.200 millones de yuan en el 2000 a 355.600 millones de yuan en el 2003. Las transacciones entre empresas representan más del 97\% del volumen del comercio electrónico. Según la Comisión Estatal de Economía y Comercio en el 2002, el 84\% de las empresas tienen sitios web públicos establecidos. Las encuestas muestran que la difusión de información sobre nuevos productos y servicios en china en la actualidad es de $(73,5 \%)$, la distribución de noticias $(70,5 \%)$, la recolección de datos de los clientes Cepal - Documentos de proyectos Tecnología de información y las comunicaciones (TIC) para el fomento de las pymes exportadoras en América. (48,9\%), los servicios de posventa $(25,4 \%)$ y la recepción de pedidos $(20,9 \%)$ son las cinco principales aplicaciones de los sitios web. A las ventas, compras, suministro y otras actividades comerciales en línea les corresponden el $16,8 \%$, el $12,1 \%$ y el $7,7 \%$, respectivamente. Los sitios Web hoy en día se utilizan para promover la imagen de la empresa. Solo un número limitado de empresas $(1,05 \%)$ ha instrumentado plenamente el comercio electrónico e inclusive no son muchas las que lo han instrumentado en forma parcial $(16,48 \%)$ según Cepal - Documentos de proyectos Tecnología de información y las comunicaciones (TIC). 


\begin{tabular}{|l|l|}
\hline Internacional & Nacional \\
\hline $\begin{array}{l}\text { - Convención y Acuerdo Operacional de la Organización Internacio- } \\
\text { nal de Satélites Marítimos (Inmarsat) } 1979 .\end{array}$ & $\begin{array}{l}\text { Constitución de la República Bolivariana de Venezuela. Art } 108 . \\
\text { Ley Orgánica de Espacios Acuáticos e insulares. }\end{array}$ \\
$\begin{array}{l}\text { - SOLAS (Convenio internacional para la seguridad de la vida huma- } \\
\text { na) Capitulo IV. }\end{array}$ & $\begin{array}{l}\text { Ley de Comercio Marítimo. Ley de Navegación } \\
\text { - Resolución MSC.202(81) (adoptada el } 19 \text { de mayo de 2006) En- } \\
\text { mienda Cap. V del SOLAS }\end{array}$ \\
$\begin{array}{l}\text { - Regla 19-1, Cap. V del SOLAS, Obligatorio cumplimiento. } \\
\text { - Sistema de Identificación y Seguimiento de Largo Alcance de los } \\
\text { Buques (LRIT). }\end{array}$ & Ley Orgánica de la Administración Publica. Art 12 \\
- Conferencia diplomática del 2002-Resolución 10 OMI. & \\
\hline
\end{tabular}

Tabla 1. Bases legales del comercio electrónico y el uso de las plataformas tecnológicas en Venezuela con relación a las empresas navieras y puertos.

Fuente. elaboración propia.

Políticas, funciones y estructura de La Convención de la Organización Internacional de Satélites Marítimos: debido a la alta proporción del comercio mundial que depende de los barcos, los satélites pueden mejorar significativamente los servicios de comunicaciones en aquellos, ante la necesidad de una organización internacional dedicada a hacer posibles estos servicios de manera indiscriminada, empleando la tecnología necesaria para ello. Por otra parte, el Acuerdo Operacional describe los aspectos financieros, técnicos y operacionales. La materialización de Inmarsat, auspiciada por la Organización Marítima Internacional (IMO), supone un esfuerzo considerable para superar controversias y dificultades, más sin embargo, siempre predominan los intereses comunes y el espíritu de acuerdo.

Estructura y funciones de los órganos de la organización: la organización tiene tres órganos: La Asamblea, El Consejo y La Dirección General.

1 La Asamblea está formada por representantes de todos los países miembros, cada uno de los cuales tiene un voto. Se reúne una vez cada dos años para revisar las actividades y los objetivos de Inmarsat y para hacer recomendaciones al Consejo.
2 El Consejo está formado por representantes de los 18 signatarios con mayor participación en la compañía, además de otros cuatro elegidos por la Asamblea para supervisar los intereses y asegurar la participación de los miembros más recientes, $\mathrm{y}$, por tanto, con menor influencia en la organización. Se reúne al menos tres veces al año. Cada miembro tiene un voto proporcional a su participación en la organización. Supervisa las actividades de la Dirección General.

3 En la Dirección General se encuentra el cuerpo permanente de Inmarsat, que comprende a unas 500 personas de 50 nacionalidades diferentes trabajando bajo la supervisión del Director General. Desarrollando las actividades rutinarias de Inmarsat.

Inmarsat y las comunicaciones mundiales: en cuanto a la descripción de las comunicaciones móviles vía satélite, es evidente que cerca del $90 \%$ de la superficie de la tierra, está fuera del alcance del cableado y de las redes de telecomunicaciones convencionales, por lo que se hace necesaria la utilización de un sistema de satélites que facilite las comunicaciones a los seres humanos que vivan, trabajen o viajen por lugares remotos de la tierra. 
Razón por la cual debemos tener en cuenta, que más del 50\% de los humanos a pesar de la globalización y el surgimiento de nuevas plataformas y herramientas tecnológicas nos encontramos "incomunicados", bien sea por la posición geográfica donde nos encontremos que en muchas oportunidades se nos hace difícil el acceso a los servicios de telecomunicaciones.

Es por esa justa razón que aparece Inmarsat, permitiendo fomentar enlaces de alta velocidad tanto en operaciones de minería remotas, hasta el simple hecho de poder controlar las maniobras de rescate en caso de algún accidente marítimo; en decir, tanto Inmarsat como otras organizaciones que proveen comunicaciones globales vía satélite desarrollan las bases para la planificación y el crecimiento económico de todo el mundo. Además, con el lanzamiento de su tercera generación de satélites, que incorpora lo último en comunicaciones digitales y tecnología de punta, así como el desarrollo del proyecto ICO de telefonía móvil vía satélite, Inmarsat permitirá establecer comunicaciones desde cualquier punto de la superficie de nuestro planeta tierra.

Del total de los 79 países que componen Inmarsat, diez corresponden a Hispanoamérica y a los países del Caribe por lo que la inversión que representan estos países dentro de la organización es del $2.5 \%$ y cerca del $15 \%$ de todos los terminales de Inmarsat que se encuentran en esta región; además, Argentina, Brasil, Colombia, Cuba y México también han invertido directamente en la compañía ICO (encargada de desarrollar un proyecto de telefonía móvil vía satélite - Inmarsat P-). Por otra parte, se espera que esta región contribuya con un $12 \%$ del mercado mundial de estos servicios, de ahí su importancia. Estos países en la actualidad disponen de 22 asientos en el Consejo, presidido recientemente por Artur Schechtman, de la empresa Embratel de Brasil. Aparte de todo esto, hay que señalar los acuerdos de cooperación de Inmarsat con: a) Aseta, Grupo Andino de Telecomunicaciones, compuesto por 5 naciones, b) Comtelca, Confederación de países centroamericanos en telecomunicaciones, con 6 naciones, c) Unión de Telecomunicaciones del Caribe, compuesto por 13 naciones. Estos acuerdos van, desde recomendaciones a seguir en materia de normalización y política de telecomunicaciones, hasta asistencia técnica y programas de desarrollo.

El capítulo IV del convenio SOLAS se refiere al uso de: radiotelegrafía y radiotelefonía: este capítulo se divide en cuatro partes, la parte A prescribe el tipo de instalaciones radioeléctricas que han de llevarse a bordo, y la parte B las prescripciones operacionales relativas a los servicios de escucha; en la parte $C$ se detallan las prescripciones técnicas. Esta última parte incluye disposiciones técnicas relativas a los radiogoniómetros y a las instalaciones radiotelegráficas para botes salvavidas a motor, junto con las correspondientes al aparato radioeléctrico portátil para embarcaciones de supervivencia. Las obligaciones del oficial radio telegrafista respecto de las anotaciones obligatorias en el registro radioeléctrico quedan consignadas en la parte $\mathrm{D}$.

El Sistema mundial de socorro y seguridad marítima (SMSSM): establece el conjunto de procedimientos de seguridad, equipos y protocolos de comunicación diseñados para aumentar la seguridad y facilitar la navegación y el rescate de embarcaciones en peligro. Este sistema está regulado por el Convenio internacional para la protección de la vida humana en el mar (SOLAS), autorizado por la Organización Marítima Internacional (OMI), organismo dependiente de la $\mathrm{ONU}$, entró en vigencia y en funcionamiento en los buques mercantes y de pasaje desde el año 1999. El GMDSS se compone de diversos sistemas, algunos de los cuales son nuevos, pero la mayoría llevan operando varios años. El sistema trata de llevar a cabo las siguientes operaciones: alerta (incluyendo posición), coordinación de búsqueda y rescate, localización (posicionamiento), provisión de información marítima, comunicaciones generales y comunicaciones de puente a puente. Los requerimientos de radio dependen del área de operación del buque más que de su tipo o tonelaje. El sistema posee mecanismos de alerta re- 
dundantes y fuentes específicas de alimentación de emergencia.

La Regla 19-1 / Cap. V SOLAS establece:

- Obligatoriedad de transmitir información LRIT

- Derechos y obligaciones de los gobiernos contratantes

- Salvaguardas entre los derechos de los Estados y las disposiciones del derecho marítimo internacional

Por lo que en este punto podemos decir que la tecnología no se puede reducir a un conjunto de diseños o planos que definen un producto; por el contrario, consiste en actividades orientadas a la solución de problemas, que incorporan formas tácitas de conocimiento mediante procesos individuales o institucionales.

Aplicación de la Regla 19-1 / Cap. V SOLA: la regla se aplicará a los siguientes tipos de buques dedicados a viajes internacionales, a) Buques de pasaje, incluidas las naves de gran velocidad, b) Buques de carga, incluidas las naves de gran velocidad, de 300 toneladas de arqueo bruto o superior y/o c) Unidades móviles de perforación mar afuera.

Los buques estarán equipados con un sistema capaz de transmitir automáticamente información LRIT de la siguiente manera:

1.- Los buques construidos durante o después del 31 de diciembre de 2008;

2.- Los buques construidos antes del 31 de diciembre de 2008 y con certificado para operar en:

\begin{tabular}{|l|l|}
\hline Área marítima & $\begin{array}{l}\text { A más tardar en la primera } \\
\text { inspección de comunicaciones } \\
\text { después de la instalación }\end{array}$ \\
\hline - A1 (Y equipados con AIS). & - No requiere cumplimiento. \\
- A1 Y A2. & - 31 de diciembre de 2008. \\
$-A 1, A 2$ Y A3. & -31 de diciembre de 2008 \\
$-A 1, A 2, A 3$ Y A4 & -1 de julio de 2009 \\
\hline
\end{tabular}

Tabla 2 .

Fuente. elaboración propia.

El sistema GMDSS divide todos los mares en cuatro áreas de navegación. Según el área en el cual opere el buque deberá llevar a bordo algunos o todos los equipos del sistema GMDSS.

1 Zona A1: es aquella zona que está bajo la cobertura de una estación en tierra que posea cobertura total en VHF DSC canal 70.

2 Zona A2: es aquella zona que está bajo la cobertura de una estación en tierra que posea cobertura total en MF DSC en la frecuencia de socorro de $2.187,5 \mathrm{Khz}$.

3 Zona A3: es aquella zona que está bajo la cobertura de una estación en tierra que posea cobertura total de los satélites de comunicaciones Inmarsat. Aproximadamente entre $\operatorname{los} 70^{\mathrm{a}}$ norte y $\operatorname{los} 70^{\mathrm{a}}$ sur.

4 Zona A4: es aquella zona que no está comprendida en ninguna de las zonas anteriores. Actualmente sería por encima de los $70^{\circ}$ norte y por debajo de los $70^{\circ}$ sur (zonas polares).

El LRIT: es un sistema de seguimiento remoto mediante el cual, un Estado miembro, a través de su Servicio de Aplicación y de su Centro de Datos, debe ser capaz de obtener posiciones actualizadas de sus buques cada 6 horas (o en intervalos inferiores hasta un mínimo de 15 minutos, si fuera necesario).

Los buques deberán transmitir automáticamente la siguiente información LRIT:

- La identidad del buque 
- La posición del buque (latitud y longitud)

- La fecha y hora de la posición

Sistema y equipamiento LRIT:

1. Equipo de a bordo de transmisión de información LRIT

2. Proveedores de servicios de somunicaciones (CSP)

3. Proveedores de servicios de aplicaciones (ASP)

4. Centros de datos LRIT (CD)

5. Plan distribución datos LRIT (DDP)

6. Intercambio internacional de datos (IDE)

7. Usuarios de datos LRIT

8. Coordinador LRIT

\section{Recibir información LRIT:}

Estado de bandera: la administración tendrá derecho a recibir información LRIT sobre los buques que enarbolan su pabellón independientemente del lugar en que estos buques puedan estar ubicados.

Estado rector del puerto: un gobierno contratante tendrá derecho a recibir información LRIT sobre los buques que han manifestado su intención de entrar en una instalación portuaria o un lugar bajo la jurisdicción de ese gobierno contratante, independientemente del lugar en que estos buques puedan estar ubicados, siempre y cuando no estén situados dentro de las aguas interiores de otro gobierno contratante.

Estado ribereño: un gobierno contratante tendrá derecho a recibir información LRIT sobre los buques con derecho a enarbolar el pabellón de otros gobiernos contratantes, que no tienen intención de entrar en una instalación portuaria o un lugar bajo la jurisdicción de ese gobierno contratante, y que naveguen dentro de una distancia no superior a 1.000 millas náuticas de su costa, siempre y cuando:
1 Tales buques no estén ubicados dentro de las aguas interiores de otro gobierno contratante.

2 Dichos buques no estén ubicados dentro del mar territorial del gobierno contratante cuyo pabellón tenga el buque derecho a enarbolar, y, la administración, a fin de preservar la seguridad u otros problemas, ha decidido no entregar la información LRIT a los gobiernos contratantes, sobre los buques que enarbolan su pabellón.

Negar información LRTI: los sistemas y equipos utilizados para cumplir los requisitos de esta regla deberán ser capaces de ser apagados abordo o ser capaces de cesar la transmisión de información LRIT cuando; A) por acuerdos internacionales, reglas o normas para la protección de la información de navegación, B) en circunstancias excepcionales y por la duración más corta posible, que el capitán considere que la operación del equipo pueda comprometer la seguridad o la protección del buque. En tal caso, el capitán deberá informar a la administración y asentará los motivos de tal decisión e indicara el período durante el cual el sistema o el equipo estuvo apagado.

En cuanto a la Constitución de la República Bolivariana de Venezuela, textualmente reza en su Artículo 108: "La República favorecerá la integración económica latinoamericana. A este fin se procurará coordinar recursos y esfuerzos para fomentar el desarrollo económico y aumentar el bienestar y seguridad comunes". Es en este punto donde vemos la disposición del Estado de forma jurídica de fomentar a través de la práctica del internacionalismo los mecanismos de integración que solo se pueden lograr en este mundo cambiante y cada vez más globalizado mediante el uso de las tecnologías de la información y comunicaciones.

Por otra parte, el Artículo 12 de la antigua ley orgánica de la administración pública ya derogada se refiere a que: "La actividad de la Administración Pública se desarrollará con base 
en los principios de economía, celeridad, simplicidad administrativa, eficacia, objetividad, imparcialidad, honestidad, transparencia, buena fe y confianza. Asimismo, se efectuará dentro de parámetros de racionalidad técnica y jurídica. La simplificación de los trámites administrativos será tarea permanente de los órganos y entes de la Administración Pública, así como la supresión de los que fueren innecesarios, todo de conformidad con los principios y normas que establezca la ley correspondiente. A fin de dar cumplimiento a los principios establecidos en esta Ley, los órganos y entes de la Administración Pública deberán utilizar las nuevas tecnologías que desarrolle la ciencia, tales como los medios electrónicos, informáticos y telemáticos, para su organización, funcionamiento y relación con las personas. En tal sentido, cada órgano y ente de la Administración Pública deberá establecer y mantener una página en la Internet, que contendrá, entre otra información que se considere relevante, los datos correspondientes a su misión, organización, procedimientos, normativa que lo regula, servicios que presta, documentos de interés para las personas, así como un mecanismo de comunicación electrónica con dichos órganos y entes disponible para todas las personas vía Internet". Que luego fue modificado según Decreto $N^{\circ}$ 6.217, con Rango, Valor y Fuerza de Ley Orgánica de la Administración Pública. - Véase N 5.890 Extraordinario de la Gaceta Oficial de la República Bolivariana de Venezuela, 31 de julio de 2008: “Mecanismos tecnológicos - Artículo 11: Los órganos y entes de la Administración Pública deberán utilizar las tecnologías que desarrolle la ciencia, tales como los medios electrónicos o informáticos y telemáticos, para su organización, funcionamiento y relación con las personas. Cada órgano y ente de la Administración Pública deberá establecer y mantener una página en Internet, que contendrá entre otra, la información que se considere relevante, los datos correspondientes a su misión, organización, procedimiento, normativa que lo regula, servicios que presta, documentos de interés para las personas, ubicación de sus dependencias e información de contactos".
Otro instrumento complementario es el Artículo 22 de la Ley Orgánica de Ciencia, Tecnología e Innovación, la cual nos señala lo siguiente: “El Ministerio de Ciencia y Tecnología coordinará las actividades del Estado que, en el área de tecnologías de información, fueren programadas. Asumirá competencias que, en materia de informática, ejercía la Oficina Central de Estadística e Informática, así como las siguientes:

1. Actuar como organismo rector del Ejecutivo Nacional en materia de tecnologías de información.

2. Establecer políticas en torno a la generación de contenidos en la red, de los órganos y entes del Estado.

3. Establecer políticas orientadas a resguardar la inviolabilidad del carácter privado y confidencial de los datos electrónicos obtenidos en el ejercicio de las funciones de los organismos públicos.

4. Fomentar y desarrollar acciones conducentes a la adaptación y asimilación de las tecnologías de información por la sociedad.

\begin{tabular}{|l|l|}
\hline Internacional & Nacional \\
\hline $\begin{array}{l}\text { SOLAS (Convenio interna- } \\
\text { cional para la seguridad de } \\
\text { la vida humana) Capítulo } \\
\text { IV. }\end{array}$ & $\begin{array}{l}\text { - Constitución de la República } \\
\text { Bolivariana de Venezuela. }\end{array}$ \\
& $\begin{array}{l}\text { - Ley Orgánica de Espacios Acuáti- } \\
\text { cos e insulares. } \\
\text { - Ley de Comercio Marítimo. } \\
\end{array}$ \\
\hline
\end{tabular}

Tabla 3.

Fuente. elaboración propia. 


\section{Transporte marítimo y modernidad}

Durante la Primera Revolución Industrial (17601860), se da el nacimiento de la industria textil, la máquina a vapor, la invención de la locomotora, dándose las primeras innovaciones tecnológicas que impulsarían respectivamente al transporte marítimo en cuanto al sistema de propulsión se refiere. De esta forma se da la evolución de la sustitución de la vela por el vapor a finales del siglo XVIII, por el ingeniero James Watt. En 1807 Fulton construye, el "Clermont" primer buque de vapor movido por ruedas de paletas que realizó su viaje desde New York hasta Albany en 32 horas. En 1819, el Savannah, utilizando un medio de propulsión mixto: la vela y el vapor, efectuó un viaje de 27 días de navegación, zarpando desde Savannah y atracando en Liverpool, lo que también es otro indicativo de la evolución del sistema marítimo (Pinacho, 1996) En la segunda mitad del siglo XIX, se fabrican los motores de explosión, de esta forma los trenes y naves empiezan a moverse con máquinas diésel, a finales del siglo XIX, con lo que comienza la segunda Revolución Científica Tecnológica y comienzan a surgir las primeras líneas regulares de cargas.

Este tipo de prestación de servicios origina la desaparición de la industria armadora, dando paso a la constitución de grandes compañías navieras, según Pinacho (1996), el detonante del uso del petróleo y su poder económico generado por sus productos derivados fue una inspiración para la construcción de un nuevo tipo de embarcación llamado buque petrolero, cuya tecnología fue puesta en servicio en 1886 mediante la puesta en funcionamiento del buque Gluckauf, de 3.020 toneladas de peso muerto, con medios de propulsión mixtos de vapor y vela. Durante la etapa de la modernidad se llevaron a cabo algunas experiencias científicas y tecnológicas que han permitido que el transporte marítimo siga evolucionando a tal punto de convertirse en una herramienta indispensable para el intercambio comercial y cultural en esta etapa del internacionalismo global.

\section{Posmodernidad y perspectivas complejas del transporte maritimo}

Los sistemas de transporte internacional se han posicionado estratégicamente como fuente principal para el traslado de mercancías y personas, más del $90 \%$ del comercio mundial de mercancías, se transporta por la vía marítima, distribución que es similar para Venezuela ya que somos altamente dependientes del comercio exterior en la exportación del petróleo e importador de otros rubros para el consumo humano ante la escasez y la falta de producción, para ser más preciso en el sector alimentos.

El predominio del contenedor y los conceptos de logística, entrega "justo a tiempo", "puerta a puerta", entre otros, han incrementado los servicios intermodales, haciendo una necesidad el multimodal-ismo, es decir un solo operador denominado Operador de Transporte Multimodal por sus siglas OTM que se hace responsable por toda la cadena de transporte desde su origen inicial hasta su destino final.

La visión planteada sólo será posible a través de la ejecución de una estrategia integrada entre los componentes de transporte marítimo, puertos, política marítima portuaria comunitaria, fortalecimiento de las administraciones marítimas, capacitación y formación y la preservación de las costas y espacios marinos.

Es fundamental agregar que actualmente los buques se diseñan, construyen, operan y tripulan con normas más estrictas que nunca. Esto ha significado consecuentemente, que la gente de mar como componente fundamental de este sistema, atraiga la atención de los organismos marítimos especializados, como factor de seguridad. La contratación de marinos requiere del cumplimiento de una serie de estándares relacionados con la seguridad de la vida humana, los buques, las cargas y el medio ambiente. 
Estos requerimientos están contenidos en la Convención Internacional sobre Estándares de Formación, Titulación y Guardia para la Gente de Mar (STCW por sus siglas en inglés), de la Organización Marítima Internacional, dirigida a garantizar la competencia y profesionalismo de la gente de mar, de quien depende la seguridad de la vida en el mar, la protección del ambiente marino y el $90 \%$ del comercio mundial. Este convenio es de obligatorio cumplimiento a partir del año 2002. Es necesario desarrollar propuestas que conlleven a instaurar un sistema eficiente, efectivo y ágil, capaz de producir marinos y profesionales marítimos de excelente calidad, asegurando así su colocación en las diferentes compañías navieras, especialmente en la industria de cruceros y en las demás ramas marítima portuaria.

Finalmente, como consecuencia del nuevo entorno internacional del transporte marítimo y del sistema portuario, esta estrategia delineará las acciones institucionales y legales pertinentes que contribuyan al desarrollo y fortalecimiento de las administraciones marítimas y del sub-sector marítimo portuario de los países, de manera que sean efectivas en los cambios que se requieren en la legislación, seguridad marítima y en la protección del medio marino ya que con algunas excepciones las administraciones marítimas no han evolucionado al mismo ritmo para atender estos temas.

\section{Conclusiones}

En la actualidad se deben desarrollar, fortalecer y consolidar las administraciones marítimas de los Estados pertenecientes a la OMI, de forma acorde con los procesos de modernización de los Estados en ejecución donde se hallan y establecerlas donde no las hay. En este sentido, se debe fortalecer la estructura orgánica, el marco jurídico y el recurso humano de las mismas. En esta medida, se debe tomarlas en cuenta a la hora de elaborar el plan orgánico anual de presupuesto, para que de esta forma se les permita adquirir nuevas plataformas tecnológicas que sirvan para darle mantenimiento a los recursos materiales y humanos idóneos necesarios, que hacen efectivo el cumplimiento de sus responsabilidades como Estado ribereño, de pabellón y de puerto. Ratificar los convenios internacionales de derecho público y privado pendientes de ratificación e implementar y administrar efectivamente los mismos.

El sector público debe utilizar las TIC, lo que en el caso de Venezuela, es necesario para el fiel cumplimiento de la leyes mencionadas anteriormente en dicho trabajo, ya que de esta forma, existirá una mayor planificación a la hora de ejecutar las políticas de desarrollo económico y social a nivel de gobierno nacional, regional y local para que de esta manera el gobierno de turno, independientemente del partido político que sea, logre ser más eficiente en cuanto al mejoramiento de su gestión interna. En el caso de las políticas de dirección general de inteligencia de mercadeo y promoción del comercio, tanto el sector público como el privado deben trabajar de forma articulada buscando las mejores soluciones informáticas para hacer cumplir los procesos de instrumentación de los acuerdos de libre comercio multilaterales, regionales y bilaterales, así como los esfuerzos unilaterales para promover el comercio mediante los acuerdos de integración que se adecuen al bienestar del país en materia social y de desarrollo económico.

En términos generales, se puede afirmar que los aspectos económicos y tecnológicos son la base para el intercambio de capitales, bienes y servicios que generan una sostenibilidad en los procesos de planificación, para las políticas y las estrategias de inversión al transporte marítimo, mediante estrategias de acción que evitan operaciones ineficientes en el transporte de carga, como viajes vacíos, utilización de modos de transporte menos contaminantes; la mejora de la infraestructura, la logística y las operaciones en el cambio de fuentes energéticas y tecnológicas de combustible limpias (menos contaminantes).

Ante los cambios mundiales, los gobiernos no deben mantener el statu quo, hay que tomar deci- 
siones y algunas de ellas muy sensibles. Incluso, podrían verse tentados a simplemente modernizar y ampliar sus facilidades con la esperanza de atraer volumen. Sin embargo, la modernización de la infraestructura de transporte y de los equipos sólo es una parte de la solución. Toda la cadena de transporte que sirve al comercio exterior debe ser mejorada e integrada, dándole más importancia a la organización y administración de las operaciones portuarias, ya que los servicios a la carga deben hacerse más eficientes para ser competitivos a nivel mundial. Entendiéndose que la confianza de los usuarios está basada mayoritariamente en un rápido tiempo de rotación, costos de servicio razonables, $y$, sobre todo, seguridad de funcionamiento.

En este sentido, la participación del sector privado es relevante, solo si se permite dentro de un marco de apertura económica que genere condiciones de mercado en los servicios que se brindan. Para ello es necesario promover la competencia, lo cual evitará males mayores como los monopolios y oligopólios, bien sean privados o por parte del Estado, con ideologías extremistas tanto capitalistas como socialistas, dándole espacio a las leyes de oferta y demanda, ganancias y pérdidas, economías de escala, autonomía gerencial, libertad para entrar y salir del mercado y una cultura enfocada al usuario.

La experiencia muestra que estos objetivos son más fáciles de alcanzar con la iniciativa privada que con las empresas públicas. Por último como reflexión quisiera acotar que en el caso de Venezuela, el Gobierno Bolivariano ha expropiado todas las empresas navieras y se ha apropiado de los puertos y aeropuertos entregándoselos al centralismo y haciendo que estos en la actualidad pierdan sus condiciones de infraestructura y la calidad de confianza de los clientes por la falta de mantenimiento de la superestructura, el conocimiento y las improvisaciones en materia de calidad de gerencia en cuanto al tema se refiere.

La suspensión de Venezuela del Mercosur, aplicada en función de las acciones del gobierno de Nicolás Maduro, es un llamado para el inmediato inicio de un proceso de transición política y restauración del orden democrático, tal como lo señala el comunicado del Mercosur, suscrito tras una reunión de los cancilleres de los Estados miembros en Sao Paulo en agosto de 2017. Esta es la segunda vez que el Mercosur, fundado en 1991, aplica esa cláusula suscrita en 1998 en Ushuaia (Argentina), y ratificada y ampliada en 2011, que estipula que "la plena vigencia de las instituciones democráticas es condición esencial" para la integración regional. En la práctica, la decisión cambia poco y nada la situación de Venezuela en el grupo, ya que el país caribeño se encuentra suspendido del Mercosur desde diciembre de 2016 por incumplir obligaciones comerciales con las que se comprometió cuando se incorporó al bloque en 2012. El único precedente de la aplicación del "Protocolo de Ushuaia " se aplicó en 2012 contra Paraguay, tras la destitución del presidente Fernando Lugo.

\section{Referencias}

Agencia Venezolana de Noticias. (2012). Tribunal del Mercosur rechaza petición para dejar sin efecto suspensión de Paraguay. Disponible en: http://www.avn.info.ve/node/123649

Arbuet, H. (2009). Claves jurídicas de la integración. Buenos Aires: Editores Rubinzal Culzoni.

Asociación Latinoamericana de Integración. (2012). Documentos. Disponible en: http://www.aladi.org/

Banco Mundial. (2013). Documentos. Disponible en: http://www.bancomundial.org/

Balestrini, M. (2008). ¿Cómo se elabora el proyecto de investigación? Séptima Edición. Caracas: Consultores Asociados Servicio Editorial. 
Balsadúa, R. (1999). El Mercosur y el derecho de la integración. Trabajo Especial de Grado presentado para optar al grado académico de Especialista en Comercio Marítimo Internacional, mención Derecho Marítimo. Caracas: Dirección de Investigación de Postgrado de la Universidad Experimental Nacional Marítima del Caribe.

Boet, E. (2013). La libre competencia en el transporte marítimo. Sexta Edición. Barcelona: J.M. Bosch Editor.

Burbano, F. (2001). Globalización, comercio e integración en América Latina. Trabajo Especial de Grado no publicado, Caracas: Universidad Metropolitana.

Cámara Argentina de Comercio. (2011). Publicaciones. Disponible en: http://www.cac.com.ar/

Castillo, M. (1983). Materiales utilizados en la construcción naval. Caracas

Comisión de Comercio de la Organización Mundial del Comercio. (2017). Régimen Especial Común para la Industria Naval. Disponible en: http://www.wto.org/indexsp.htm

Comisión Económica para América Latina y el Caribe. (2008). Estudio económico de América Latina y el Caribe. Disponible en: http://www. eclac.cl/noticias/paginas/8/33638/PPTEE08_ final.pdf

Comunidad Andina de Naciones. (2012). Documentos. Disponible en: http://www.comunidadandina.org/index.aspx

Conferencia de las Naciones Unidas UNCTAD (2013). Informe de la Secretaria de la UNCTAD. Nueva York y Ginebra: Organización de Naciones Unidas

Conferencia de las Naciones Unidas UNCTAD (2012). El transporte marítimo: informe de la Secretaria de la UNCTAD. Nueva York y Ginebra: Organización de Naciones Unidas.
Conferencia de las Naciones Unidas UNCTAD (2008). El transporte marítimo: informe de la Secretaria de la UNCTAD. Nueva York y Ginebra: Organización de Naciones Unidas.

Conferencia de las Naciones Unidas UNCTAD (1999). Informe de la Secretaria de la UNCTAD. Nueva York y Ginebra: Organización de Naciones Unidas.

Del Arenal, C. (2009). Introducción al estudio de las relaciones internacionales, Tercera edición. Madrid: Editorial Tecnos.

García, Josefina. Genua Gladis. (s.f.). Acuerdos económicos internacionales: una guía para la gerencia. Caracas: Ediciones IESA.

González, R. (2008). Venezuela en la encrucijada de la globalización. Revista SIC - Centro Gumilla.

Herrera, P. (2010). La exención tributaria. Madrid: Editorial Colex.

Hilbert, M. (2008). La sociedad de la información en América Latina y el Caribe. Santiago de Chile, inédito.

Hoffmann, J. (2009). El papel de los puertos y del transporte marítimo en América Latina. Documento de trabajo presentado en el Comité de Puertos de la Comunidad Andina. Disponible en: http:/eclac.cl/transporte/perfil/tendencias.pdf

Instituto de Investigación Económica Aplicada (IPEA). (2014). Infraestructura económica en Brasil: diagnósticos y perspectivas para el 2025, Libro 6 / Volumen 1. Brasilia: Secretaría de Asuntos Estratégicos de la Presidencia de la República de Brasil.

Martínez, J.M. (2009). Enciclopedia general del mar. Barcelona: Ediciones Garriga.

Márquez, Y. (2010). Los incentivos fiscales y el desarrollo del sector marina mercante en la 
República Bolivariana de Venezuela y en los Estados parte del Mercosur, período 2000 2008. Trabajo Especial de Grado presentado para optar al grado académico de Magíster en Transporte Marítimo. Caracas: Dirección de Investigación de Postgrado de la Universidad Experimental Nacional Marítima del Caribe.

Mercosur. (1991). Tratado de la Asunción. Disponible en: http://www.mercosur.int/innovaportal/file/719/1/CMC_1991_TRATADO_ES_ Asuncion.pdf

Montero, M. y Hochman, E. (2005). Investigación documental. Técnicas y procedimientos. Caracas: Editorial Panapo.

Omaña, Gustavo. (2008). El nuevo régimen de beneficios fiscales en el sector acuático nacional. Disponible en: https://docs.google.com/document/pub? id=1beKiG46aI-cdIF_FCzQuPnamre2TE9zx

Organización de Países Exportadores de Petróleo. (2012). Publicaciones. Disponible en: http:// www.opec.org/opec_web/en/

Organización Mundial del Comercio. (2013). Documentos y recursos. Disponible en: http:// www.wto.org/indexsp.htm

Revista de Intereses Marítimos. (2013). Marina mercante. Disponible en: http://www.ara.mil. ar/archivos/Docs/IIMM-06-Capitulo\%206. pdf

Revista de Transporte Marítimo de la UNCTAD. (2013). Archivo. División de Tecnología y Logística y la secretaría de la UNCTAD. Disponible en: http://www.marcotradenews.com.

Regas, R. (2010). Gran atlas universal. Barcelona: Editorial Sol 90.

Rodrigo, O., Masatsugu, Y. \& Yasushi, U. (2003). Tecnología de la información y las comunicaciones (TIC) para el fomento de las pymes ex- portadoras en América Latina y Asia oriental. Cepal (LC/W 27).

Salama, R. (2009). Influencia de la globalización sobre las líneas navieras de servicio regular en la República Bolivariana de Venezuela. Trabajo Especial de Grado mención publicación. Caracas: Universidad Nacional Experimental Marítima del Caribe.

Salomón, L. (2007). El régimen de participación del capital extranjero para el registro de buques en Venezuela. Trabajo Especial de Grado presentado para optar al grado académico de Especialista en Comercio Marítimo Internacional, mención Derecho Marítimo. Caracas: Dirección de Investigación de Postgrado de la Universidad Experimental Nacional Marítima del Caribe.

Sánchez, M. (2012). Análisis del Decreto con Rango y Valor y Fuerza de Ley Orgánica de los Espacios Acuáticos 2008. Revista Doctum, (14), 109131.

Sánchez, R. (2012). Transporte Marítimo Internacional. ajustes, efectos y las lecciones de la mitología. Boletín Marítimo, (51). Unidad de Recursos Naturales e Infraestructura, Cepal.

Sánchez, R. (2009). Los desafíos del sistema de transporte en los países de América del Sur. Cepal Serie 149. (LC/L 3013-P).

Sellone, E. (2007). Estudio comparado de la regulación y régimen de la responsabilidad civil en el transporte multimodal caso: Venezuela, CAN y Mercosur, período 2002-2007. Trabajo Especial de Grado presentado para optar al grado académico de Magíster en Transporte Marítimo. Caracas: Dirección de Investigación de Postgrado de la Universidad Experimental Nacional Marítima del Caribe.

Silva, D. (2007). Tendencias del transporte marítimo de contenedores en el eje norte - sur en los albores del siglo XXI. Trabajo Especial de Gra- 
do presentado para optar al grado académico de Magíster en Transporte Marítimo. Caracas: Dirección de Investigación de Postgrado de la Universidad Experimental Nacional Marítima del Caribe.

Silva, D. (2005). El transporte marítimo un análisis desde la perspectiva de la complejidad. Revista Doctum, 8(1), 47-60.

SOLAS (1974). Convenio Internacional para la Seguridad de la Vida Humana en el Mar. OMI. Disponible en: http://www.cndh.org.mx/DocTR/2016/JUR/A70/01/JUR-20170331-II44.pdf

Pinacho, J. (1996). Tráfico marítimo. Madrid. Fondo Editorial de Ingeniería Naval/Colegio Oficial de Ingenieros Navales.

Reyes, José (2014). Relación entre los incentivos fiscales y el comportamiento del sector de la marina mercante de los Estados partes del Mercosur durante los años 2008 - 2013. Trabajo Especial de Grado presentado para optar al grado académico de Magíster en Transporte Marítimo. Caracas: Dirección de Investigación de Postgrado de la Universidad Experimental Nacional Marítima del Caribe.

Tamames, R. (2008). Estructura económica internacional. Madrid: Alianza.

Uckmar, V., Altamirano, A. \& Taveira, H. (2008). Impuestos sobre el comercio internacional. Buenos Aires: Marcial Pons.

Universidad Experimental Marítima del Caribe. (2010). Manual para la elaboración, presentación, defensa y aprobación de trabajos de grado. Vicerrectorado Académico. Dirección de Investigación y Postgrado. Catia La Mar: Universidad Experimental Marítima del Caribe.

Universidad Pedagógica Experimental Libertador (2010). Manual de trabajos de grado de especialización y maestría y tesis doctorales. Caracas: Fedupel. 